Gazi University
Journal of Science
http://dergipark.gov.tr/gujs

\title{
Bayesian Confidence Interval Estimation of Weibull Modulus Under Increasing Failure Rate
}

\author{
Meryem YALCINKAYA ${ }^{1, *}$ (D) Burak BIRGOREN $^{2}$ (D) \\ ${ }^{1}$ Hitit University, Rectorate, 19030, Corum, Turkey \\ ${ }^{2}$ Kirikkale University, Department of Industrial Engineering, 71451, Kirikkale, Turkey
}

\section{Highlights}

- Two Bayesian models were proposed to estimate the confidence interval of the Weibull modulus.

- The Weibull modulus is an important problem in the fracture strength modeling.

- The Bayesian models were based on the prior of increasing failure rates of all most materials.

- This prior argument requires the Weibull modulus to be more than 1 due to wear-out failure.

\begin{tabular}{l} 
Article Info \\
\hline \\
Received: $12 / 05 / 2020$ \\
Accepted: $15 / 07 / 2020$ \\
Keywords \\
Weibull modulus \\
Bayesian \\
Interval estimation \\
Increasing failure \\
Rate
\end{tabular}

\section{INTRODUCTION}

Weibull distribution [1] is a well-established characterization tool in the field of modeling failure times and analyzing the mechanical properties of ceramic and composite materials, such as fracture strength of ceramics, metallic matrix composites and ceramic matrix composites [2], flexural strength of brittle materials [3], fracture toughness behavior of steels in ductile-brittle transition region [4].

The two-parameter Weibull distribution is generally recommended for characterizing ceramic and composite material properties [4]. Let $T$ denote a Weibull random variable modeling the strength of a material. Then $T$ has the following probability density function with parameters $\sigma_{0}$ and $m$ :

$$
f(t)=\frac{m}{\sigma_{0}}\left(\frac{t}{\sigma_{0}}\right)^{m-1} e^{-\left(\frac{t}{\sigma_{0}}\right)^{m}}
$$

where $\sigma_{0}>0$ and $m>0$ are the scale and shape parameters, respectively. The shape parameter is alternatively referred to as the Weibull modulus. Weibull modulus is used as a measure of the variability of the strength of materials [4] or as a measure of component reliability and system reliability [5].

\begin{abstract}
Estimating the confidence interval of the Weibull modulus is an important problem in the fracture the sample size is small due to high experimental costs. For this purpose, several classical estimating confidence lower bounds for Weibull percentiles using the prior knowledge that the due to wear-out failure. In this study, under the same prior information, two Bayesian Weibull models, one using the same prior argument and the other a relaxed version of it, have been performances have been compared against the maximum likelihood method with Monte Carlo simulations. The results show that the Bayesian Weibull models significantly outperform the maximum likelihood method for almost all Weibull modulus and sample size values.
\end{abstract}


The cumulative distribution function, $F(t)$, giving the probability of fracture at a stress level $t$ is expressed as Equation (2), where reliability function $R(t)=e^{-\left(\frac{t}{\sigma_{0}}\right)^{m}}$.

$$
\mathrm{P}(T \leq t)=F(t)=1-R(t)=1-e^{-\left(\frac{t}{\sigma_{0}}\right)^{m}}
$$

In practice, high experimental costs limit the number of samples to be tested; and in general, as the sample size decreases, the statistical inferences for Weibull modulus $(m)$ becomes worse, because the precision and the biasing (the coefficient of variation) of the Weibull estimators increase as the sample size decreases for any method [6-8], In addition, Khalili and Kromp [9] showed the coefficient of variation as a function of the inverse square root $\left(1 / n^{1 / 2}\right)$. Therefore, for small sample sizes, new estimation methods or models with better statistical inferences are needed to be investigated.

In general, Bayesian methods have superior performance over classical ones for small samples. There are many studies on the estimation of the Weibull modulus $(m)$ using classical and Bayesian inference methods; however Bayesian studies in the materials science literature are very limited. A previous study [6] proposed a Bayesian estimation model for confidence intervals of Weibull lower percentiles specifically designed for material science applications, and showed that it outperforms alternative classical methods for small sample sizes. The Bayesian model defines a prior distribution suitable for material science applications and generally applicable to any brittle material without any specific knowledge. The basic prior argument in the study is that ceramic and composite materials have increasing failure rates, which requires $m$ to be greater than 1 .

In reliability analysis increasing failure rates typically refer to the wear-out period in a bathtub curve, indicating late life of a product. Usually decreasing and constant failure rates are also observed in earlier periods in a product's life cycle. The random variable $T$ is the failure time in such product-life cycle applications. In materials science applications, however, $T$ denotes strength measurements. For increasing failure rates, the fracture probability increases by increasing pressure levels of $t$, which is normally expected for all brittle materials. Theoretical studies in materials science show that strength distribution of carbon fibers and fibrous composite materials exhibit increasing failure rates [10-12], and Yalcinkaya and Birgoren [13] discusses why increasing failure rates should also apply to ceramics: Increasing failure rates require $m>1$, which explains the lower limit of the uniform prior. As for the upper limit, a value of 100 indicates an amount of variability that is negligibly small for most practical purposes. Estimated values for existing materials also support this upper limit: For metals such as aluminum and steel, and metal alloys, $m$ is around $100[14,15]$. For traditional ceramics (e.g. brick, pottery, chalk etc), $m$ is between 1 and 3 [14, 15]; for engineered ceramics (e.g. SiC, Al2O3, Si3N4) it is between 5 and $10[14,15]$.

Motivated by this recent result [13], the two Bayesian models have been proposed, one using the same prior argument and the other a relaxed version of it, for confidence interval estimation of $m$ and their performances have been assessed against the maximum likelihood method, which is the most frequently used one among classical estimation methods. The first Bayesian model uses a Uniform prior distribution with limits $(1,100)$ for $m$ and the non-informative Jeffrey's prior for the Weibull scale parameter $\left(\sigma_{0}\right)$. In theory, $m$ values can range from 0 to $\infty$, but experimental and theoretical studies in materials science support these uniform prior limits. The first model strictly constrains $m$ in the interval $(1,100)$, which may be argued to be valid for most materials, but certainly not all materials. The second model assigns a nonzero probability $1-\alpha$ to the event that $m$ is in $(1,100)$. A prior distribution can be elicited for $m$ by following the steps of a maximal entropy hash (MEH) prior elicitation method, which will be explained in later. MEH priors encompass the standard maximal entropy (ME) priors and the maximal data information (MDI) priors in order to increase their chances of being proper [16]. For $\sigma_{0}$, the non-informative Jeffrey's prior has been used, as in the first Bayesian model.

In the first step of this study, credible interval (for Bayesian inference) and confidence interval (for classical inference) estimation algorithms have been developed based on the maximum likelihood (MLE) method and the two Bayesian Weibull (BW) models (using Uniform $(1,100)$ \& Jeffrey's priors and MEH \& 
Jeffrey's priors). In the second step, Monte Carlo simulations have been designed and run in the C++ language for the comparisons with large simulation run numbers. Small sample sizes between 3 and 20 have been used in the simulations.

In the literature, many authors have proposed various estimation methods for the Weibull parameters and their confidence intervals for complete and censored data. The handbooks [17-22] provide a recent detailed discussion of classical and Bayesian methods. MLE [20], Linear Least Squares (LLS) [21], and Weighted Linear Least Squares (WLLS) [22-24] are among the popular classical estimation methods. The most common estimation method is the MLE; see Nelson [25], Johnson et al. [26], Murthy et al. [27], and Dodson [19] for a detailed discussion.

In the materials science literature, statistical inferences of the estimators and confidence intervals for $m$ have been studied extensively using classical methods [2-6, 9, 20-24, 28-37]. Among these studies, Gong [31] introduced a new probability index for point and interval estimation of $m$ for ceramics with an LLS method. In addition, McCool and Phan [38] proposed a simple method based on Menon's original work [39] for estimating exact confidence intervals of $m$ and characteristic strength. McCool [40] developed a program for creating pivotal tables according to user-entered percentage and sample volume information for point and interval estimation of $m$ and percentiles. Bütikofera et al. [41] compared point estimates and 95\% confidence intervals for $m$ by two LLS methods. They called conventional LLS methods as YonX methods, and proposed the XonY method by interchanging the axes in the least squares analysis.

While there are major differences among classical methods, Bayesian methods basically use the same formulation, as will be discussed later; they differ only by the choice of the prior distribution of the parameters $[17,18]$. In the general scientific literature, there are many Bayesian point estimation studies on $m$, see for example [42-47]; however Bayesian interval estimation studies are limited, see Aron et al. [48] as an example. The details of Bayesian Weibull analysis can be found in [49]. In the materials science literature, there is only one study on Bayesian interval estimation for the Weibull modulus: Simoa et al. [50] proposed a new Bayesian method for determination of strength and crack growth using fatigue data from previous tests as prior information. The remaining studies in materials science are generally based on point estimation of $m$ for multiple purposes, such as investigating size effect of materials under different operational conditions [51] or understanding the statistical behavior of the strength of materials [52].

In this study, the two proposed BW models do not require any past data, and make use of the information that the Weibull modulus is more than 1 and less than 100, which is considered a proper upper value for brittle materials. MEH priors developed by Bousquet [16] encompass the ME and the MDI priors for eliciting a proper prior. In Bayesian inference, the ME and the MDI priors have been studied by Zellner [53-55], Berger [56] and Soofi [57], among many others. The interested reader is also referred to Skilling [58], Zellner [55], Le Besnerais et al. [59], Soofi [60], Miller and Yan [61].

\section{MATERIAL METHOD}

\subsection{Estimation of Confidence Bounds for Weibull Modulus}

The Weibull modulus, $m$, has been recently used as a measure of the variability of the strength, the mechanical reliability or the probability of failure of materials [62-64]. A higher Weibull modulus $(m>$ 20) indicates a narrower distribution of failure strengths, and, therefore, a more reliable material such that failure occurs in a more predictable and controlled manner. A material characterized by a low Weibull modulus has a broad distribution of failure strengths and exhibits low predictable failure behavior. Since Weibull modulus characterizes the shape of the failure probability curve, it is used as a strength reliability parameter in materials science and has to be estimated accurately for performing a successful reliability analysis.

Weibull modulus can be estimated using classical and Bayesian inferences. For both inferences, the estimates of $m, \widehat{m}$, can be quite unreliable, especially in small samples. Therefore, instead of $\widehat{m}$ values, confidence bounds for $m$ have been used for the characterization of mechanical properties [62-64]. 
Confidence intervals are called credible intervals in the parlance of Bayes' theory. Credible intervals of parameters and functions of parameters are calculated from posterior distributions in Bayesian inference [65].

\subsection{Maximum Likelihood Method}

The maximum likelihood estimation (MLE) is a method of estimating the parameters of a probability distribution by maximizing a likelihood function. Using the density of $f(t)$, as defined in Equation (1), the likelihood function for the Weibull distributed independent data observations $T_{1}, T_{2}, \ldots, T_{n}$ can be written as

$$
L\left(m, \sigma_{0}\right)=L\left(t_{1}, t_{2}, \ldots, t_{n}, m, \sigma_{0}\right)=\prod_{i=1}^{n} f\left(t_{i} ; m, \sigma_{0}\right)=m^{n} \sigma_{0}^{n} t_{1}^{\sigma_{0}-1} t_{2}^{\sigma_{0}-1} \ldots t_{n}^{\sigma_{0}-1} e^{-m \sum_{i=1}^{n} \sigma_{i}^{\sigma_{0}}} .
$$

The likelihood function in Equation (3) can be maximized by taking logarithms of it (Equation (4)), differentiating with respect to $m$ and $\sigma_{0}$ and equating to zero, $d l\left(m, \sigma_{0}\right) / d m=0$ and $d l\left(m, \sigma_{0}\right) / d \sigma_{0}=0$ :

$$
l\left(m, \sigma_{0}\right)=\operatorname{InL}\left(m, \sigma_{0}\right)=n \operatorname{In} m-n \operatorname{In} \sigma_{0}+(n-1) \sum_{i=1}^{n} \operatorname{In} \frac{t_{i}}{\sigma_{0}}-\sum_{i=1}^{n} \operatorname{In}\left(\frac{t_{i}}{\sigma_{0}}\right)^{m} .
$$

The estimating equations maximizing $l\left(m, \sigma_{0}\right)$ are shown in the set of Equation (5) and (6) and the solution set of these equations is called the maximum likelihood estimator of $\hat{m}$ ve $\hat{\sigma}_{0}$ [66]

$$
\begin{aligned}
& \frac{n}{\widehat{m}}-n \operatorname{In} \hat{\sigma}_{0}+\sum_{i=1}^{n} \operatorname{In}\left(t_{i}\right)-\sum_{i=1}^{n}\left(\frac{t_{i}}{\hat{\sigma}_{0}}\right)^{\widehat{m}} \operatorname{In}\left(\frac{t_{i}}{\hat{\sigma}_{0}}\right) \\
& \hat{\sigma}_{0}=\left(\frac{\sum_{i=1}^{n}\left(t_{i}\right)^{\widehat{m}}}{n}\right)^{1 / \widehat{m}}
\end{aligned}
$$

where $t_{1}, t_{2}, \ldots, t_{n}$ are an observed sample of size $n$. The Newton-Raphson method is usually employed for solving Equation (5) for $\widehat{m}$. Then, $\hat{\sigma}_{0}$ is found by substituting $\widehat{m}$ into Equation (6). Thoman [67] showed that $\widehat{m} / m$ is a pivotal variable, that is, independent of $\sigma_{0}$ and $m$. The distribution of $\widehat{m} / m$ can be found for a given $n$ and $\alpha$ value by Monte Carlo simulation. Since the pivotal statistic $\widehat{m} / m$ has the same distribution with $m, \widehat{m}$ can be used instead of $\widehat{m} / m$ in Equation (7) and then Equation (8) is obtained

$$
\begin{aligned}
& P\left(d_{1-\alpha / 2}<\frac{\widehat{m}}{m}<d_{\alpha / 2}\right)=1-\alpha \\
& P\left(\frac{\widehat{m}}{d_{\alpha / 2}}<m<\frac{\widehat{m}}{d_{1-\alpha / 2}}\right)=1-\alpha .
\end{aligned}
$$

The upper and lower bounds, respectively $d_{1-\alpha / 2}$ and $d_{\alpha / 2}$, are obtained by simulation of $\widehat{m}$.

\subsection{The Bayesian Weibull (BW) Models}

Bayesian estimation inference makes use of one's prior knowledge about the parameters as well as the useable data. When any prior knowledge about the parameter is not available, it is possible to make use of the non-informative priors in Bayesian analysis [45]. In this article, Bayesian inference is applied to estimating the parameters of Weibull distribution assuming a prior knowledge about $m$ exists. 
Applying Bayes' rule [68] on the 2-parameter Weibull distribution and assuming the prior distributions of $m$ and $\sigma_{0}$ are independent, the following posterior distribution is expressed as

$$
P\left(m, \sigma_{0} \mid \boldsymbol{T}\right)=\frac{L\left(m, \sigma_{0}\right) P(m) P\left(\sigma_{0}\right)}{\int_{0}^{\infty} \int_{0}^{\infty} L\left(m, \sigma_{0}\right) P(m) P\left(\sigma_{0}\right) d \sigma_{0} d m}
$$

where $P\left(m, \sigma_{0} \mid \boldsymbol{T}\right)$ is the posterior distribution of $m$ and $\sigma_{0}, L\left(m, \sigma_{0}\right)=\mathrm{P}\left(\boldsymbol{T} \mid m, \sigma_{0}\right)=\prod_{i=1}^{n} f\left(t_{i} ; m ; \sigma_{0}\right)$ is the likelihood function of the data given $m$ and $\sigma_{0}, P(m)$ and $P\left(\sigma_{0}\right)$ are the prior distributions of $m$ and $\sigma_{0}$, respectively. The double integrals in Equation (9) have no closed forms, since Weibull shape and scale parameters are treated as random variables having prior distributions. Thus, to get the estimators analytically based on the formulations is impossible, and some numerical integration methods, such as trapezoid rule and Simpson's rule, are needed to calculate the estimators [49]. For more information about numerical integration methods, see "Applied Mathematics for Science and Engineering", Chapter 4 "Numerical Quadrature" [69]. In this study, trapezoid integration rule was used for solving the double integrals.

After deriving the posterior distribution $P\left(m, \sigma_{0} \mid \boldsymbol{T}\right)$, the marginal posterior probability $P(m \mid \boldsymbol{T})$ of $m$ is determined by summing the posterior probabilities across the alternative values for $\sigma_{0}$ and the credible interval of $m$ is calculated based on $P(m \mid \boldsymbol{T})$. At a given confidence level $a$, the two-sided credible intervals can be calculated by solving Equation (10) for $m$ with trapezoid integration rule:

$$
\int_{m_{\text {lower }}}^{m_{\text {upper }}} P(m \mid \boldsymbol{T}) d m=\int_{m_{\text {lower }}}^{m_{\text {upper }}} \int_{0}^{\infty} P\left(m, \sigma_{0} \mid \boldsymbol{T}\right) d \sigma_{0} d m=1-\alpha .
$$

If the marginal posterior density of $m, P(m \mid \boldsymbol{T})=\int_{0}^{\infty} P\left(m, \sigma_{0} \mid \boldsymbol{T}\right) d \sigma_{0}$, is unimodal, then for a given value of $\alpha$, the shortest credible interval for $m$ is given by $C I=\{m: P(m \mid \boldsymbol{T}) \geq k\}$ where $k$ is the largest number so that ;

$$
\int_{\{m: P(m \mid \boldsymbol{T}) \geq k\}} P(m \mid \boldsymbol{T}) d m=\int_{\{m: P(m \mid \boldsymbol{T}) \geq k\}} \int_{0}^{\infty} P\left(m, \sigma_{0} \mid \boldsymbol{T}\right) d \sigma_{0} d m=1-\alpha .
$$

The credible set described in Equation (11) is called a highest posterior density interval (HPD), as it consists of the values of the parameter for which the posterior density is the highest. An HPD interval has the shortest width among all other credible intervals. When the marginal distribution of the interest of parameter is not symmetric, a $100(1-\alpha) \%$ HPD interval is more desirable. In this context, the HPD interval is more appropriate for $m$ of which the marginal posterior distribution has been proven to be not symmetric by the simulations in this study. For a recent detailed discussion of HPD credible intervals, see [70].

\subsection{Priors and Algorithms for the BW Models}

As mentioned before, using the prior argument based on increasing failure rates of materials, two Bayesian Weibull models have been proposed for credible intervals of $m$ in this study.

\section{BW1 Model: $m \sim$ Uniform $(1,100) \& \sigma_{0} \sim$ Jeffrey's prior}

The first Bayesian model (BW1) in Equation (12) assumes that the Weibull modulus has a uniform prior on the interval of $(1,100)$ as discussed before. It is also assumed that there is no prior knowledge about the scale parameter, so the Jeffrey's prior is chosen for $\sigma_{0}$ as it is non-informative, using only the information in a sample. The uniform distribution implies that any Weibull modulus value is equally likely in the interval $(1,100)$. An expert may specify a narrower interval, and even change the distribution. However, in materials testing, whereabouts of the Weibull modulus can hardly be known before actually conducting the tests. Because, in cases of where a Weibull material has such characteristic properties as relative flaw 
density, fracture origins, internal stress fields, gradients, toughness etc., a deviation from Weibull behavior is identified and so $m$ becomes stress-dependent [71]. An interval of $(1,100)$, on the other hand, will be valid for most, if not all, ceramics and composite materials, and hence eliminate any need for an expert opinion for a specific material.

$$
f\left(m, \sigma_{0} \mid T\right)==\frac{\prod_{i=1}^{n} f\left(t_{i} ; m ; \sigma_{0}\right) \operatorname{Unf}(m ; 1,100) 1 / \sigma_{0}}{\int_{0}^{\infty} \int_{0}^{\infty} \prod_{i=1}^{n} f\left(t_{i} ; m ; \sigma_{0}\right) \operatorname{Unf}(m ; 1,100) 1 / \sigma_{0} d \sigma_{0} d m}
$$

\section{BW2 Model: $m \sim M E H\left(\gamma_{0} \cong 0.70\right) \& \sigma_{0} \sim$ Jeffrey's prior}

A uniform distribution on the $(1,100)$ interval imposes strong constraints for the prior probability of $m$ : the probability that $m<1$ and $m>100$ is zero. The second Bayesian model (BW2) assumes $P(1<m<100)=1-\alpha$, which provides a relaxation. Under this assumption, an MEH prior distribution is elicitied by the MEH elicitation method. MEH prior encompasses the ME priors and the MDI priors when there is no prior information or when little expert knowledge is considered only. The MEH prior distribution of $m$ is given in Equation (13);

$$
P_{M E H}(m)=m^{-1} \exp \left(-\gamma_{0} \gamma / m\right) / \Gamma^{\gamma_{0}}(1 / m) \quad, \quad \gamma_{0}>0, m>0
$$

where $\gamma_{0}$ is the shape parameter, $\gamma$ is the Euler constant $(\gamma \cong 0.57721)$ and $\Gamma($.$) is the gamma function.$

In the first step for the MEH elicitation method, an expert gives a range for $m$ under a probability $1-\alpha$ and the value of $\gamma_{0}$ is searched to satisfy Equation (14). In the second step, the MEH prior distribution is elicited by replacing Equation (13) with the result value of $\gamma_{0}$. In addition, by combining descent methods (such as Newton-Raphson) and Monte Carlo methods, numerous MEH prior distributions for the Weibull modulus can be derived, assessing a range of values for $\gamma_{0}$. See [16] for a detailed discussion of MEH prior and elicitation method:

$$
P\left(m_{\text {low }}<m<m_{\text {up }}\right)=\frac{\int_{m_{\text {low }}}^{m_{u p}} m^{-1} \exp \left(-\gamma_{0} \gamma / m\right) / \Gamma^{\gamma_{0}}(1 / m) d m}{\int_{0}^{\infty} m^{-1} \exp \left(-\gamma_{0} \gamma / m\right) / \Gamma^{\gamma_{0}}(1 / m) d m}=1-\alpha .
$$

In this study, $\left[m_{\text {low }}, m_{u p}\right]=[1,100]$. However, no parameter value for $\gamma_{0}$, can be found for high probabilities such 0.80 and 0.90 for this interval. This is a disadvantage of this prior distribution, while it is very practical for its simple, one-parameter construct. In this study, a high value for $1-\alpha$ is desirable, thus, a value of $\gamma_{0}$ is searched to maximize $1-\alpha$ in Equation (14). The Figure 1 is obtained by plotting Equation (14) for different $\gamma_{0}$ values for the $(1,100)$ interval. A maximum probability of $1-\alpha=0.66$ is obtained for $\gamma_{0} \cong 0.70$. Therefore, the desired MEH prior is as follows: As in BW2, the Jeffrey's prior is chosen for $\sigma_{0}$ :

$$
P_{M E H}(m)=m^{-1} \exp (-0.7 \gamma / m) / \Gamma^{\gamma_{0}}(1 / m) .
$$

After obtaining $P_{M E H}(m)$, the BW2 model has the following posterior distribution function:

$$
f\left(m, \sigma_{0} \mid T\right)=\frac{\prod_{i=1}^{n} f\left(t_{i} ; m ; \sigma_{0}\right) m^{-1} \exp (-0.7 \gamma / m) / \Gamma^{\gamma_{0}}(1 / m) 1 / \sigma_{0}}{\int_{0}^{\infty} \int_{0}^{\infty} \prod_{i=1}^{n} f\left(t_{i} ; m ; \sigma_{0}\right) m^{-1} \exp (-0.7 \gamma / m) / \Gamma^{\gamma_{0}}(1 / m) 1 / \sigma_{0} d \sigma_{0} d m} .
$$




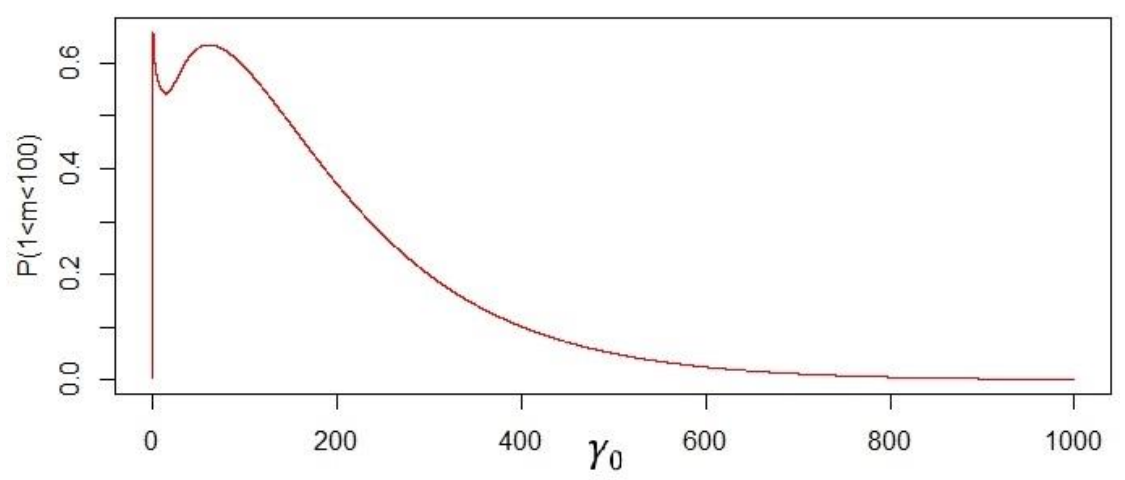

Figure 1. $P(1<m<100)$ for different $\gamma_{0}$ values

\section{MONTE CARLO SIMULATION STUDY}

\subsection{Simulation Inputs}

An extensive Monte Carlo simulation has been performed to compare the performances of the MLE, BW1 and BW2 models. Figure 2 shows the flowchart of the simulation procedure implemented in $\mathrm{C}++$.

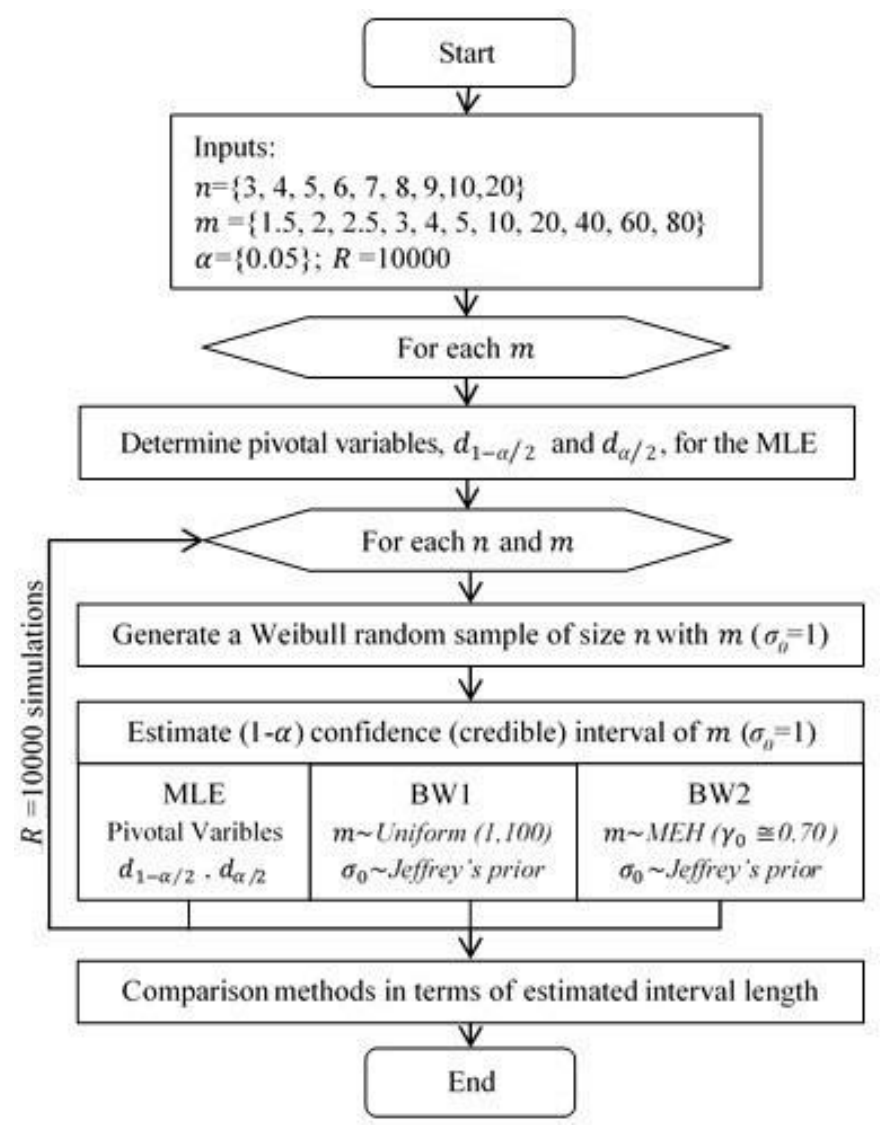

Figure 2. Flow chart of the simulation procedure

In the simulation, $\sigma_{0}$ is a fixed at $\sigma_{0}=1$, since it has no effect on the results. For each set of given sample size and the prescribed Weibull modulus, $R=10000$ intervals are estimated using the methods described in the experimental section. The reason for selecting the small $m$ values in Figure 2 is to examine the effect of the shape of the Weibull probability density function, which is positively skewed for $m<2.6$, approximates the normal pdf for $2.6<m<3.7$ and is negatively skewed for $m>3.7$ [49]. 


\subsection{Comparison Criterion: Median Interval Length}

In this study, median interval length is chosen for comparing the performances of MLE and BW methods. The median statistic is more representative of the central tendency due to the asymmetry of the distribution of $\widehat{m}$ for most classical methods [9]. For Bayesian methods, the obtained $\widehat{m}$ distributions in this study have verified the existence of asymmetry. However, median interval lengths cannot be compared without considering simulation errors. The replication number $R=10000$ is quite a large number considering computationally expensive Bayesian methods; yet, it still produces a significant simulation error. Simulation errors will be quantified by constructing confidence intervals for the median interval length. Let $Z_{1}, \ldots, Z_{R}$ be estimated interval lengths from a simulation of $R$ replications for a particular $n$ and $m$. Also let $Z_{(1)} \leq \cdots \leq Z_{(R)}$ denote the associated order statistics, and $\tilde{Z}$ denote the associated sample median. A 95\% confidence interval for the true median $\tilde{\mu}_{Z}$ is $\left(Z_{\left(l_{1}\right)}, Z_{\left(u_{1}\right)}\right)$, where [72]

$$
l_{1}=\left[(0.5) R-1.96 \sqrt{(0.5)^{2} R}+0.5\right] \& u_{1}=\left[(0.5) R+1.96 \sqrt{(0.5)^{2} R}+1.5\right] .
$$

\subsection{Simulation Results}

A detailed summary of the simulation results is presented in Tables 1-2 for each $n$ and $m$ according to the 95\% confidence intervals of $\tilde{\mu}_{Z}$ in Equation (17). The results are also shown graphically in Figures 3 and 4. In Table 3, for each $n$ and $m$, the result of the best method, that is the shortest interval length, is shown in bold and the second best method is shown underlined and italic. In some cases, two methods are shown to be best methods at the same time due to intersecting confidence intervals, which indicates that there is no statistically significant difference between the true medians $\left(\tilde{\mu}_{Z}\right)$. 
Table 1. Estimated Interval Lengths for Weibull Modulus $(m)$ for $n=3,4,5,6$ and 7

\begin{tabular}{|c|c|c|c|c|c|c|c|}
\hline \multirow[b]{2}{*}{$n$} & \multirow[b]{2}{*}{$m$} & \multicolumn{2}{|l|}{ MLE } & \multicolumn{2}{|l|}{ BW1 } & \multicolumn{2}{|l|}{ BW2 } \\
\hline & & $\tilde{Z}$ & $95 \%$ CI of $\tilde{\mu}_{Z}$ & $\tilde{Z}$ & $95 \%$ CI of $\tilde{\mu}_{Z}$ & $\tilde{Z}$ & $95 \%$ CI of $\tilde{\mu}_{Z}$ \\
\hline 3 & 1.5 & 3.720 & $(3.701,3.738)$ & 3.557 & $(3.512,3.622)$ & 2.910 & $(2.867,2.951)$ \\
\hline 3 & 2 & 4.978 & $(4.952,5.001)$ & $\overline{4.852}$ & $(4.776,4.946)$ & 3.838 & $(3.789,3.894)$ \\
\hline 3 & 2.5 & 6.185 & $(6.156,6.218)$ & $\overline{6.261}$ & $(6.149,6.372)$ & 4.799 & $(4.735,4.868)$ \\
\hline 3 & 3 & 7.462 & $(7.426,7.498)$ & $\overline{7.646}$ & $(7.511,7.736)$ & 5.715 & $(5.624,5.795)$ \\
\hline 3 & 4 & $\overline{9.913}$ & $(9.857,9.963)$ & 10.459 & $(10.286,10.652)$ & 7.612 & $(7.511,7.735)$ \\
\hline 3 & 5 & $\underline{12.398}$ & $(12.34,12.459)$ & 13.062 & $(12.87,13.27)$ & 9.355 & $(9.211,9.486)$ \\
\hline 3 & 10 & $\underline{24.850}$ & $(24.737,24.97)$ & 26.462 & $(26.04,26.859)$ & 18.381 & $(18.123,18.639)$ \\
\hline 3 & 20 & 49.499 & $(49.258,49.737)$ & 53.943 & $(53.013,54.783)$ & 36.991 & $(36.384,37.632)$ \\
\hline 3 & 40 & 99.411 & $(98.936,99.863)$ & 72.256 & $(72.029,72.441)$ & 71.898 & $(70.902,72.886)$ \\
\hline 3 & 60 & 148.569 & $(147.845,149.261)$ & 72.549 & $(72.378,72.747)$ & $\underline{108.077}$ & $(106.386,111.164)$ \\
\hline 3 & 80 & 199.439 & $(198.412,200.454)$ & 70.342 & $(70.17,70.499)$ & 145.418 & $(143.286,146.866)$ \\
\hline 4 & 1.5 & 2.915 & $(2.903,2.926)$ & 2.612 & $(2.566,2.65)$ & 2.446 & $(2.417,2.476)$ \\
\hline 4 & 2 & 3.895 & $(3.879,3.909)$ & $\underline{3.695}$ & $(3.642,3.75)$ & 3.297 & $(3.254,3.338)$ \\
\hline 4 & 2.5 & $\underline{4.878}$ & $(4.86,4.897)$ & 4.790 & $(4.712,4.865)$ & 4.119 & $(4.072,4.166)$ \\
\hline 4 & 3 & 5.861 & $(5.839,5.881)$ & 5.976 & $(5.911,6.06)$ & 5.014 & $(4.953,5.068)$ \\
\hline 4 & 4 & 7.772 & $(7.742,7.801)$ & 8.073 & $(7.972,8.177)$ & 6.608 & $(6.546,6.688)$ \\
\hline 4 & 5 & 9.734 & $(9.695,9.771)$ & 10.186 & $(10.093,10.303)$ & 8.305 & $(8.207,8.397)$ \\
\hline 4 & 10 & $\overline{19.510}$ & $(19.439,19.581)$ & 20.296 & $(20.059,20.568)$ & 16.541 & $(16.355,16.749)$ \\
\hline 4 & 20 & $\underline{38.835}$ & $(38.674,38.989)$ & 40.905 & $(40.506,41.385)$ & 33.349 & $(32.971,33.792)$ \\
\hline 4 & 40 & $\underline{78.164}$ & $(77.899,78.47)$ & 66.403 & $(66.166,66.663)$ & 66.475 & $(65.705,67.296)$ \\
\hline 4 & 60 & $\overline{116.602}$ & $(116.169,117.013)$ & 67.472 & $(67.29,67.68)$ & $\underline{100.313}$ & $(98.003,102.123)$ \\
\hline 4 & 80 & 155.602 & $(154.992,156.125)$ & 64.329 & $(64.089,64.517)$ & 131.289 & $(129.999,132.689)$ \\
\hline 5 & 1.5 & 2.470 & $(2.462,2.478)$ & 2.173 & $(2.152,2.202)$ & 2.182 & $(2.158,2.206)$ \\
\hline 5 & 2 & $\overline{3.289}$ & $(3.279,3.299)$ & $\underline{3.085}$ & $(3.049,3.124)$ & 2.909 & $(2.877,2.944)$ \\
\hline 5 & 2.5 & $\underline{4.126}$ & $(4.112,4.139)$ & $\underline{4.099}$ & $(4.054,4.148)$ & 3.701 & $(3.655,3.737)$ \\
\hline 5 & 3 & 4.920 & $(4.904,4.937)$ & 5.019 & $(4.974,5.07)$ & 4.429 & $(4.395,4.469)$ \\
\hline 5 & 4 & $\overline{6.592}$ & $(6.57,6.613)$ & 6.743 & $(6.682,6.798)$ & 5.894 & $(5.838,5.945)$ \\
\hline 5 & 5 & $\underline{8.213}$ & $(8.185,8.237)$ & 8.458 & $(8.366,8.551)$ & 7.365 & $(7.287,7.439)$ \\
\hline 5 & 10 & $\underline{16.441}$ & $(16.386,16.491)$ & 17.066 & $(16.93,17.271)$ & 14.982 & $(14.833,15.113)$ \\
\hline 5 & 20 & $\overline{32.963}$ & $(32.866,33.076)$ & 33.929 & $(33.648,34.293)$ & 29.666 & $(29.45,29.956)$ \\
\hline 5 & 40 & 65.823 & $(65.611,66.059)$ & $\underline{61.285}$ & $(60.915,61.583)$ & 59.558 & $(58.967,60.172)$ \\
\hline 5 & 60 & 98.623 & $(98.303,98.943)$ & 63.706 & $(63.499,63.889)$ & $\underline{90.950}$ & $(90.45,91.367)$ \\
\hline 5 & 80 & 131.510 & $(131.068,131.953)$ & 60.164 & $(59.903,60.389)$ & 118.539 & $(117.608,119.479)$ \\
\hline 6 & 1.5 & 2.185 & $(2.18,2.191)$ & 1.893 & $(1.869,1.912)$ & 1.975 & $(1.958,1.993)$ \\
\hline 6 & 2 & 2.911 & $(2.903,2.919)$ & $\underline{2.762}$ & $(2.731,2.795)$ & 2.664 & $(2.639,2.686)$ \\
\hline 6 & 2.5 & $\underline{3.647}$ & $(3.636,3.658)$ & $\overline{3.667}$ & $(3.628,3.705)$ & 3.378 & $(3.348,3.407)$ \\
\hline 6 & 3 & 4.355 & $(4.342,4.367)$ & 4.433 & $(4.386,4.48)$ & 4.030 & $(3.986,4.065)$ \\
\hline 6 & 4 & 5.812 & $(5.797,5.83)$ & 5.955 & $(5.897,6.021)$ & 5.390 & $(5.338,5.455)$ \\
\hline 6 & 5 & $\overline{7.260}$ & $(7.241,7.28)$ & 7.444 & $(7.375,7.526)$ & 6.733 & $(6.662,6.801)$ \\
\hline 6 & 10 & $\underline{14.548}$ & $(14.51,14.592)$ & 15.065 & $(14.945,15.201)$ & 13.647 & $(13.51,13.75)$ \\
\hline 6 & 20 & 29.135 & $(29.054,29.221)$ & 29.849 & $(29.618,30.096)$ & 27.115 & $(26.854,27.369)$ \\
\hline 6 & 40 & 58.194 & $(58.027,58.359)$ & $\underline{56.623}$ & $(56.325,56.895)$ & 54.436 & $(53.926,54.922)$ \\
\hline 6 & 60 & 87.460 & $(87.203,87.705)$ & $\overline{60.890}$ & $(60.684,61.101)$ & $\underline{81.105}$ & $(80.678,81.44)$ \\
\hline 6 & 80 & 116.202 & $(115.874,116.533)$ & 56.752 & $(56.536,56.974)$ & 107.413 & $(106.462,108.299)$ \\
\hline 7 & 1.5 & 1.984 & $(1.979,1.989)$ & 1.731 & $(1.712,1.747)$ & 1.838 & $(1.827,1.854)$ \\
\hline 7 & 2 & 2.639 & $(2.633,2.646)$ & 2.523 & $(2.493,2.557)$ & 2.453 & $(2.431,2.476)$ \\
\hline 7 & 2.5 & $\underline{3.310}$ & $(3.302,3.319)$ & 3.321 & $(3.295,3.35)$ & 3.098 & $(3.076,3.124)$ \\
\hline 7 & 3 & $\overline{3.970}$ & $(3.96,3.981)$ & $\overline{3.986}$ & $(3.954,4.021)$ & 3.687 & $(3.663,3.719)$ \\
\hline 7 & 4 & $\overline{5.294}$ & $(5.281,5.308)$ & $\overline{5.354}$ & $(5.311,5.402)$ & 4.943 & $(4.904,4.983)$ \\
\hline 7 & 5 & $\overline{6.613}$ & $(6.595,6.631)$ & 6.644 & $(6.599,6.699)$ & 6.134 & $(6.086,6.192)$ \\
\hline 7 & 10 & $\overline{13.215}$ & $(13.181,13.249)$ & $\overline{13.419}$ & $(13.305,13.53)$ & 12.427 & $(12.334,12.546)$ \\
\hline 7 & 20 & $\underline{26.456}$ & $(26.398,26.522)$ & 26.754 & $(26.511,26.989)$ & 24.804 & $(24.614,24.998)$ \\
\hline 7 & 40 & $\overline{52.946}$ & $(52.807,53.088)$ & $\underline{52.323}$ & $(51.964,52.697)$ & 49.760 & $(49.403,50.212)$ \\
\hline 7 & 60 & 79.275 & $(79.081,79.488)$ & $\overline{58.316}$ & $(58.108,58.518)$ & $\underline{74.215}$ & $(73.268,75.16)$ \\
\hline 7 & 80 & 105.890 & $(105.604,106.183)$ & 53.973 & $(53.752,54.189)$ & 98.197 & $(97.521,98.852)$ \\
\hline
\end{tabular}


Table 2. Estimated Interval Lengths for Weibull Modulus $(m)$ for $n=8,9,10$ and 20

\begin{tabular}{|c|c|c|c|c|c|c|c|}
\hline \multirow[b]{2}{*}{$\boldsymbol{n}$} & \multirow[b]{2}{*}{$m$} & \multicolumn{2}{|l|}{ MLE } & \multicolumn{2}{|l|}{ BW1 } & \multicolumn{2}{|l|}{ BW2 } \\
\hline & & $\tilde{Z}$ & $95 \%$ CI of $\tilde{\mu}_{Z}$ & $\tilde{Z}$ & $95 \%$ CI of $\tilde{\mu}_{Z}$ & $\tilde{Z}$ & $95 \%$ CI of $\tilde{\mu}_{Z}$ \\
\hline 8 & 1.5 & 1.824 & $(1.82,1.828)$ & 1.576 & $(1.558,1.595)$ & $\underline{1.699}$ & $(1.687,1.711)$ \\
\hline 8 & 2 & 2.438 & $(2.431,2.444)$ & $\underline{2.325}$ & $(2.305,2.35)$ & 2.270 & $(2.252,2.287)$ \\
\hline 8 & 2.5 & $\underline{3.041}$ & $(3.034,3.048)$ & 3.042 & $(3.016,3.064)$ & 2.872 & $(2.848,2.892)$ \\
\hline 8 & 3 & $\underline{3.656}$ & $(3.647,3.665)$ & $\underline{3.674}$ & $(3.645,3.704)$ & 3.438 & $(3.414,3.464)$ \\
\hline 8 & 4 & 4.868 & $(4.856,4.879)$ & 4.953 & $(4.915,4.994)$ & 4.637 & $(4.599,4.676)$ \\
\hline 8 & 5 & 6.090 & $(6.077,6.106)$ & 6.127 & $(6.079,6.177)$ & 5.744 & $(5.708,5.791)$ \\
\hline 8 & 10 & $\underline{12.188}$ & $(12.156,12.22)$ & $\underline{12.296}$ & $(12.201,12.392)$ & 11.544 & $(11.446,11.637)$ \\
\hline 8 & 20 & $\underline{24.372}$ & $(24.318,24.423)$ & $\underline{24.461}$ & $(24.269,24.649)$ & 23.011 & $(22.849,23.17)$ \\
\hline 8 & 40 & 48.682 & $(48.565,48.8)$ & $\underline{48.268}$ & $(48.002,48.563)$ & 46.179 & $(45.813,46.556)$ \\
\hline 8 & 60 & 73.083 & $(72.911,73.258)$ & 56.143 & $(55.991,56.292)$ & $\underline{69.359}$ & $(69.074,69.659)$ \\
\hline 8 & 80 & 97.440 & $(97.204,97.69)$ & 51.766 & $(51.536,51.982)$ & $\underline{92.057}$ & $(91.249,92.774)$ \\
\hline 9 & 1.5 & 1.693 & $(1.689,1.697)$ & 1.477 & $(1.462,1.493)$ & 1.599 & $(1.588,1.612)$ \\
\hline 9 & 2 & 2.256 & $(2.251,2.261)$ & $\underline{2.208}$ & $(2.189,2.227)$ & 2.151 & $(2.132,2.166)$ \\
\hline 9 & 2.5 & $\underline{2.828}$ & $(2.821,2.834)$ & $\underline{2.829}$ & $(2.81,2.85)$ & 2.688 & $(2.67,2.707)$ \\
\hline 9 & 3 & $\underline{3.389}$ & $(3.382,3.397)$ & $\underline{3.416}$ & $(3.393,3.437)$ & 3.227 & $(3.203,3.248)$ \\
\hline 9 & 4 & $\underline{4.520}$ & $(4.51,4.531)$ & 4.555 & $(4.52,4.588)$ & 4.311 & $(4.282,4.34)$ \\
\hline 9 & 5 & $\underline{5.657}$ & $(5.645,5.669)$ & $\underline{5.715}$ & $(5.668,5.75)$ & 5.396 & $(5.361,5.443)$ \\
\hline 9 & 10 & $\underline{11.292}$ & $(11.27,11.319)$ & 11.494 & $(11.4,11.57)$ & 10.904 & $(10.828,10.984)$ \\
\hline 9 & 20 & $\underline{22.601}$ & $(22.552,22.653)$ & 22.842 & $(22.666,23.002)$ & 21.688 & $(21.521,21.821)$ \\
\hline 9 & 40 & $\underline{45.097}$ & $(45,45.194)$ & $\underline{45.254}$ & $(44.975,45.619)$ & 43.201 & $(42.922,43.507)$ \\
\hline 9 & 60 & 67.735 & $(67.583,67.88)$ & 54.238 & $(54.098,54.371)$ & $\underline{66.198}$ & $(65.733,66.654)$ \\
\hline 9 & 80 & 90.484 & $(90.283,90.697)$ & 50.060 & $(49.81,50.327)$ & $\underline{85.335}$ & $(84.809,85.924)$ \\
\hline 10 & 1.5 & 1.591 & $(1.587,1.594)$ & 1.405 & $(1.392,1.417)$ & 1.520 & $(1.508,1.528)$ \\
\hline 10 & 2 & 2.125 & $(2.12,2.129)$ & $\underline{2.079}$ & $(2.063,2.093)$ & 2.023 & $(2.01,2.037)$ \\
\hline 10 & 2.5 & $\underline{2.651}$ & $(2.645,2.656)$ & $\underline{2.642}$ & $(2.625,2.659)$ & 2.522 & $(2.507,2.539)$ \\
\hline 10 & 3 & $\underline{3.183}$ & $(3.177,3.19)$ & $\underline{3.191}$ & $(3.169,3.215)$ & 3.042 & $(3.019,3.062)$ \\
\hline 10 & 4 & 4.250 & $(4.241,4.259)$ & $\overline{4.286}$ & $(4.259,4.311)$ & 4.085 & $(4.06,4.11)$ \\
\hline 10 & 5 & $\underline{5.300}$ & $(5.288,5.311)$ & 5.360 & $(5.325,5.399)$ & 5.110 & $(5.074,5.14)$ \\
\hline 10 & 10 & 10.597 & $(10.575,10.618)$ & 10.757 & $(10.674,10.826)$ & 10.267 & $(10.195,10.345)$ \\
\hline 10 & 20 & $\underline{21.164}$ & $(21.121,21.212)$ & 21.532 & $(21.387,21.667)$ & 20.556 & $(20.442,20.705)$ \\
\hline 10 & 40 & $\overline{42.485}$ & $(42.394,42.573)$ & $\underline{42.803}$ & $(42.565,43.049)$ & 40.831 & $(40.554,41.148)$ \\
\hline 10 & 60 & 63.513 & $(63.389,63.642)$ & 52.596 & $(52.472,52.712)$ & 61.186 & $(60.498,61.869)$ \\
\hline 10 & 80 & 84.785 & $(84.596,84.97)$ & 48.616 & $(48.408,48.875)$ & 80.861 & $(80.362,81.266)$ \\
\hline 20 & 1.5 & 1.073 & $(1.071,1.074)$ & 1.021 & $(1.013,1.028)$ & $\underline{1.051}$ & $(1.046,1.055)$ \\
\hline 20 & 2 & $\underline{1.430}$ & $(1.428,1.432)$ & $\underline{1.434}$ & $(1.425,1.44)$ & 1.405 & $(1.399,1.411)$ \\
\hline 20 & 2.5 & $\underline{1.787}$ & $(1.784,1.789)$ & $\underline{1.796}$ & $(1.788,1.804)$ & 1.761 & $(1.753,1.769)$ \\
\hline 20 & 3 & $\underline{2.145}$ & $(2.142,2.148)$ & $\underline{2.155}$ & $(2.144,2.164)$ & 2.112 & $(2.101,2.121)$ \\
\hline 20 & 4 & $\overline{2.859}$ & $(2.855,2.863)$ & $\overline{2.865}$ & $(2.852,2.878)$ & 2.810 & $(2.798,2.821)$ \\
\hline 20 & 5 & $\underline{3.573}$ & $(3.568,3.578)$ & $\underline{3.583}$ & $(3.566,3.601)$ & 3.517 & $(3.499,3.534)$ \\
\hline 20 & 10 & $\underline{7.145}$ & $(7.134,7.156)$ & $\underline{7.180}$ & $(7.146,7.211)$ & 7.049 & $(7.02,7.079)$ \\
\hline 20 & 20 & $\overline{14.304}$ & $(14.285,14.324)$ & 14.359 & $(14.301,14.429)$ & 14.097 & $(14.033,14.17)$ \\
\hline 20 & 40 & 28.609 & $(28.569,28.649)$ & $\underline{28.251}$ & $(28.133,28.361)$ & 27.807 & $(27.686,27.907)$ \\
\hline 20 & 60 & 42.879 & $(42.818,42.939)$ & 40.032 & $(39.899,40.178)$ & $\underline{42.039}$ & $(41.721,42.353)$ \\
\hline 20 & 80 & 57.195 & $(57.11,57.276)$ & 40.194 & $(40.075,40.299)$ & $\underline{54.416}$ & $(54.067,54.775)$ \\
\hline
\end{tabular}

As shown in Tables 1 and 2 and Figures 3 and 4, the BW2 model generally outperforms the others by yielding the smallest values for the median interval length $(\tilde{Z})$. The exceptional cases are $m=60,80$ for all $n$, and $m=1.5$ for $n>5$, where BW1 model is the best method. These are the cases where $m$ is close to the uniform prior boundaries 1 and 100. The restriction of the prior that $m$ cannot take values below 1 and above 100 is likely to induce an effect of narrowing of the interval length. Figures 3-4 show that performance of the BW1 model becomes significantly better as $m$ approaches 1 or 100 .

The MLE method is the worst method according to $\tilde{Z}$ values, however there is no statistical difference between the MLE method and the BW1 model for $2.5 \leq m \leq 20$ and all $n$, since the corresponding confidence intervals in Tables 1 and 2 intersect with each other. Also, by increasing $n$, the performances of all the three methods become closer to each other. 
a)

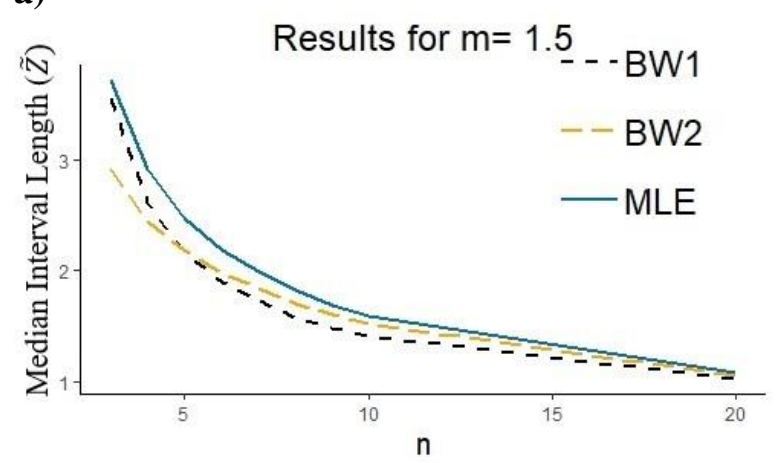

c)

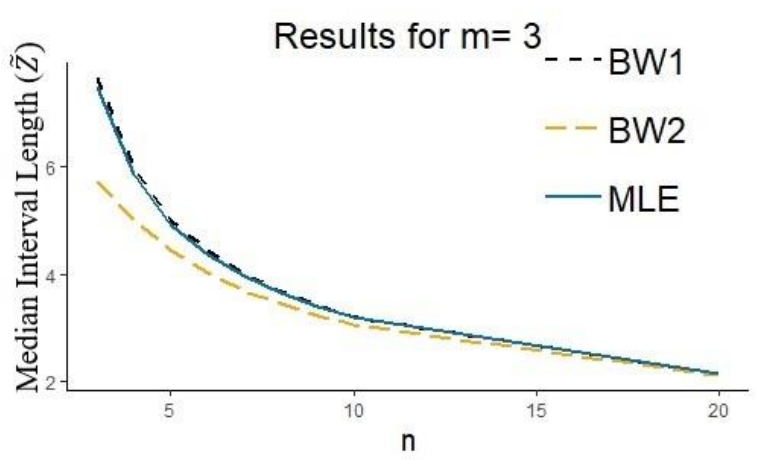

b)

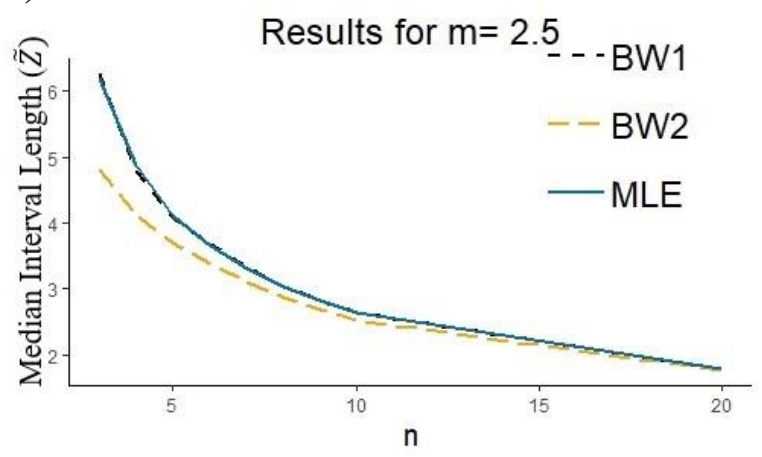

d)

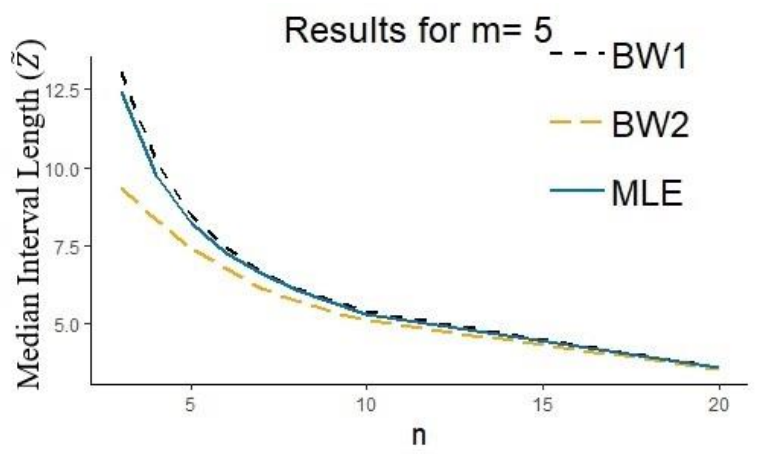

Figure 3. Expected Median Interval Lengths for (a) $m=1.5$ (b) $m=2.5$, (c) $m=3$, (d) $m=5$

a)

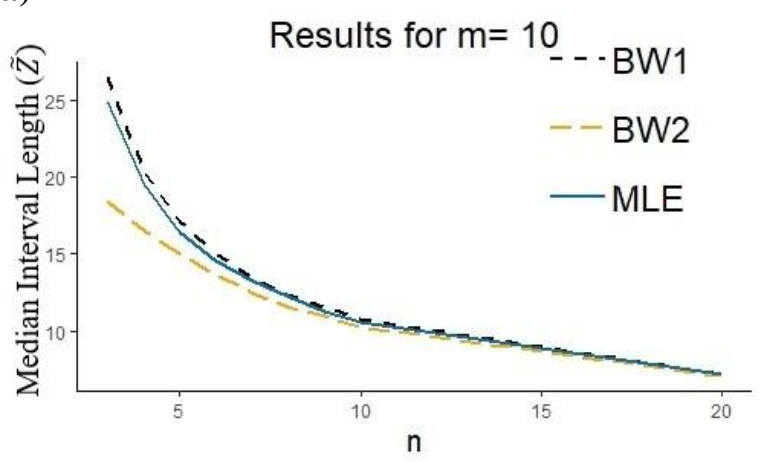

c)

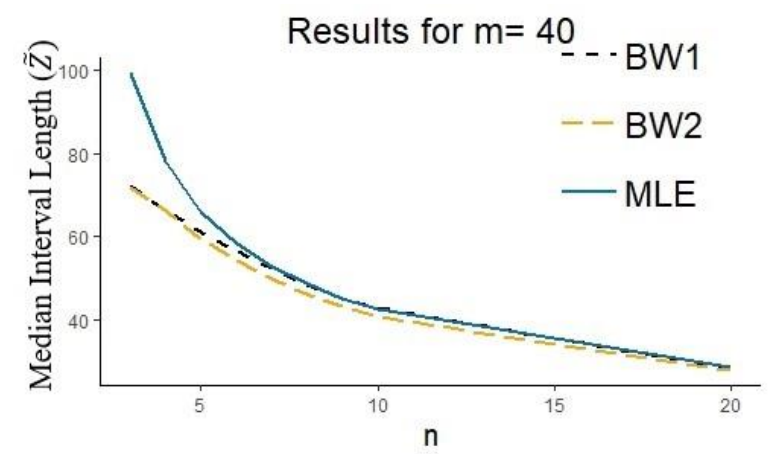

b)

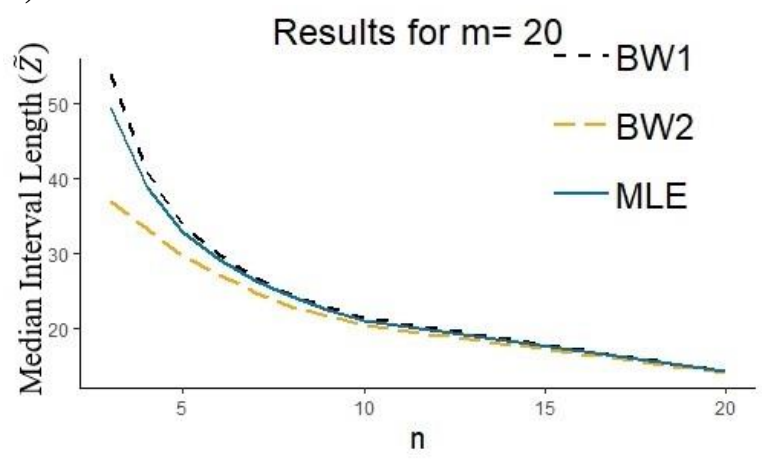

d)

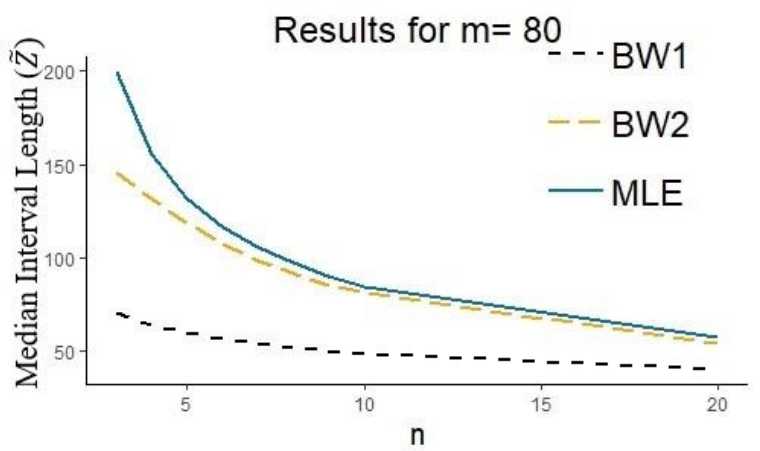

Figure 4. Expected Median Interval Lengths for (a) $m=10$ (b) $m=20$, (c) $m=40$, (d) $m=80$

Having established the superior performance of the Bayesian models, a more practical way to compare in terms of engineering costs is to calculate the relative sample sizes needed to obtain approximately the same $\tilde{Z}$ values. For this purpose, the BW2 model has been chosen as the reference method for $m<60$ since it produces the smallest values for the median interval length $(\tilde{Z})$ in the majority of the cases. Similarly, the 
BW1 model has the smallest lengths, and hence is the reference method for $m \geq 60$. Tables 3 and 4 show the results for a comparison of the three methods used where the reference method, as described above, is the method that produces the minimum sample size in each row. The sample sizes required by the other two methods that produce the same or very similar interval lengths as the reference method have been calculated and listed in the same row.

First, let's examine the graph of $m=40$ in Figure 4(c). For $n=3$, the $\tilde{Z}$ value of the BW2 model is approximately 72; and this value is achieved by the MLE method at $n=4$ and by the BW1 at $n=3$. This means that the BW1 and BW2 models show approximately the same performance, but as compared to the MLE method, a saving of (4-3)/4 $=25 \%$ is achieved by the BW2 model in testing costs for reliability analysis. This is reflected at the row with $m=40$ and the BW2 column's value at $n=3$ in Table 3 with a $0 \%$ saving as compared to the BW1 model and 25\% saving as compared to the MLE method. Similarly, as shown in Table 4, the $\tilde{Z}$ value of the BW1 model is 67 for $n=4$ and $m=60$, and this value is approximately achieved by the MLE method at $n=10$ indicating a saving of (10-4)/10 $=60 \%$ if the BW1 model were used, and is achieved by the BW2 model at $n=9$ indicating a saving of (9-4)/9 $=56 \%$ if the BW1 model were used.

According to Table 3, the percent savings achieved by using the BW2 model is at least $9 \%$ for of $3 \leq m \leq$ 40 and $n \leq 10$. For $m=1.5$, there is almost no difference between the BW1 and BW2 models for $n \leq 6$, and the BW1 model starts to perform better for $n>6$. Similarly, Table 4 indicates a minimal saving of $9 \%$ for $n \leq 20$ when the BW1 model is used. 
Table 3. Comparison of median expected lengths in terms of sample size (Ref: BW2)

\begin{tabular}{|c|c|c|c|c|c|c|c|c|c|c|c|c|c|}
\hline \multirow{2}{*}{\multicolumn{3}{|c|}{$\begin{array}{l}\text { Reference: } \\
\text { BW2 }\end{array}$}} & \multicolumn{4}{|c|}{ Equivalent $n$ to produce $\tilde{Z}$} & \multirow{2}{*}{\multicolumn{3}{|c|}{$\begin{array}{l}\text { Reference: } \\
\text { BW2 }\end{array}$}} & \multicolumn{4}{|c|}{ Equivalent $n$ to produce $\tilde{Z}$} \\
\hline & & & \multicolumn{2}{|c|}{ BW1 } & \multicolumn{2}{|c|}{ MLE } & & & & \multicolumn{2}{|c|}{ BW1 } & \multicolumn{2}{|c|}{ MLE } \\
\hline$m$ & $n$ & $\tilde{Z}$ & $n$ & $\%$ Saving & $n$ & $\%$ Saving & $m$ & $n$ & $\tilde{Z}$ & $n$ & $\%$ Saving & $n$ & $\%$ Saving \\
\hline 1.5 & 3 & 2.91 & 4 & 0.25 & 4 & 0.25 & 5 & 3 & 9.355 & 4 & 0.25 & 4 & 0.25 \\
\hline 1.5 & 4 & 2.446 & 4 & 0.00 & 5 & 0.20 & 5 & 4 & 8.305 & 5 & 0.20 & 5 & 0.20 \\
\hline 1.5 & 5 & 2.182 & 5 & 0.00 & 6 & 0.17 & 5 & 5 & 7.365 & 6 & 0.17 & 6 & 0.17 \\
\hline 1.5 & 6 & 1.975 & 6 & 0.00 & 7 & 0.14 & 5 & 6 & 6.733 & 7 & 0.14 & 7 & 0.14 \\
\hline 1.5 & 7 & 1.838 & 6 & -0.17 & 8 & 0.13 & 5 & 7 & 6.134 & 8 & 0.13 & 8 & 0.13 \\
\hline 1.5 & 8 & 1.699 & 7 & -0.14 & 9 & 0.11 & 5 & 8 & 5.744 & 9 & 0.11 & 9 & 0.11 \\
\hline 1.5 & 9 & 1.599 & 8 & -0.13 & 10 & 0.10 & 5 & 9 & 5.396 & 10 & 0.10 & 10 & 0.10 \\
\hline 1.5 & 10 & 1.52 & 9 & -0.11 & 11 & 0.09 & 5 & 10 & 5.11 & 11 & 0.09 & 11 & 0.09 \\
\hline 1.5 & 20 & 1.051 & 19 & -0.05 & 20 & 0.00 & 5 & 20 & 3.517 & 20 & 0.00 & 20 & 0.00 \\
\hline 2 & 3 & 3.838 & 4 & 0.25 & 4 & 0.25 & 10 & 3 & 18.381 & 5 & 0.40 & 4 & 0.25 \\
\hline 2 & 4 & 3.297 & 5 & 0.20 & 5 & 0.20 & 10 & 4 & 16.541 & 5 & 0.20 & 5 & 0.20 \\
\hline 2 & 5 & 2.909 & 5 & 0.00 & 6 & 0.17 & 10 & 5 & 14.982 & 6 & 0.17 & 6 & 0.17 \\
\hline 2 & 6 & 2.664 & 6 & 0.00 & 7 & 0.14 & 10 & 6 & 13.647 & 7 & 0.14 & 7 & 0.14 \\
\hline 2 & 7 & 2.453 & 7 & 0.00 & 8 & 0.13 & 10 & 7 & 12.427 & 8 & 0.13 & 8 & 0.13 \\
\hline 2 & 8 & 2.27 & 8 & 0.00 & 9 & 0.11 & 10 & 8 & 11.544 & 9 & 0.11 & 9 & 0.11 \\
\hline 2 & 9 & 2.151 & 9 & 0.00 & 10 & 0.10 & 10 & 9 & 10.904 & 10 & 0.10 & 10 & 0.10 \\
\hline 2 & 10 & 2.023 & 11 & 0.09 & 11 & 0.09 & 10 & 10 & 10.267 & 11 & 0.09 & 11 & 0.09 \\
\hline 2 & 20 & 1.405 & 20 & 0.00 & 20 & 0.00 & 10 & 20 & 7.049 & 20 & 0.00 & 20 & 0.00 \\
\hline 3 & 3 & 5.715 & 4 & 0.25 & 4 & 0.25 & 20 & 3 & 36.991 & 4 & 0.25 & 4 & 0.25 \\
\hline 3 & 4 & 5.014 & 5 & 0.20 & 5 & 0.20 & 20 & 4 & 33.349 & 5 & 0.20 & 5 & 0.20 \\
\hline 3 & 5 & 4.429 & 6 & 0.17 & 6 & 0.17 & 20 & 5 & 29.666 & 6 & 0.17 & 6 & 0.17 \\
\hline 3 & 6 & 4.03 & 7 & 0.14 & 7 & 0.14 & 20 & 6 & 27.115 & 7 & 0.14 & 7 & 0.14 \\
\hline 3 & 7 & 3.687 & 8 & 0.13 & 8 & 0.13 & 20 & 7 & 24.804 & 8 & 0.13 & 8 & 0.13 \\
\hline 3 & 8 & 3.438 & 9 & 0.11 & 9 & 0.11 & 20 & 8 & 23.011 & 9 & 0.11 & 9 & 0.11 \\
\hline 3 & 9 & 3.227 & 10 & 0.10 & 10 & 0.10 & 20 & 9 & 21.688 & 10 & 0.10 & 10 & 0.10 \\
\hline 3 & 10 & 3.042 & 11 & 0.09 & 11 & 0.09 & 20 & 10 & 20.556 & 11 & 0.09 & 11 & 0.09 \\
\hline 3 & 20 & 2.112 & 20 & 0.00 & 20 & 0.00 & 20 & 20 & 14.097 & 20 & 0.00 & 20 & 0.00 \\
\hline 4 & 3 & 7.612 & 4 & 0.25 & 4 & 0.25 & 40 & 3 & 71.898 & 3 & 0.00 & 4 & 0.25 \\
\hline 4 & 4 & 6.608 & 5 & 0.20 & 5 & 0.20 & 40 & 4 & 66.475 & 4 & 0.00 & 5 & 0.20 \\
\hline 4 & 5 & 5.894 & 6 & 0.17 & 6 & 0.17 & 40 & 5 & 59.558 & 5 & 0.00 & 6 & 0.17 \\
\hline 4 & 6 & 5.39 & 7 & 0.14 & 7 & 0.14 & 40 & 6 & 54.436 & 6 & 0.00 & 7 & 0.14 \\
\hline 4 & 7 & 4.943 & 8 & 0.13 & 8 & 0.13 & 40 & 7 & 49.76 & 8 & 0.13 & 8 & 0.13 \\
\hline 4 & 8 & 4.637 & 9 & 0.11 & 9 & 0.11 & 40 & 8 & 46.179 & 9 & 0.11 & 9 & 0.11 \\
\hline 4 & 9 & 4.311 & 10 & 0.10 & 10 & 0.10 & 40 & 9 & 43.201 & 10 & 0.10 & 10 & 0.10 \\
\hline 4 & 10 & 4.085 & 11 & 0.09 & 11 & 0.09 & 40 & 10 & 40.831 & 11 & 0.09 & 11 & 0.09 \\
\hline 4 & 20 & 2.81 & 20 & 0.00 & 20 & 0.00 & 40 & 20 & 27.807 & 20 & 0.00 & 21 & 0.05 \\
\hline
\end{tabular}

Table 4. Comparison of median expected lengths in terms of sample size (Ref: BW1)

\begin{tabular}{|c|c|c|l|l|l|l|}
\hline \multicolumn{3}{|l|}{ Reference: } & \multicolumn{3}{l|}{ Equivalent $n$ to produce $\tilde{Z}$} \\
\cline { 4 - 7 } BW1 & \multicolumn{2}{l|}{ BW2 } & \multicolumn{2}{l|}{ MLE } \\
\hline$m$ & $n$ & $\tilde{Z}$ & $n$ & \% Saving & $n$ & \% Saving \\
\hline 60 & $\mathbf{3}$ & 72.549 & 8 & 0.63 & 9 & 0.67 \\
60 & $\mathbf{4}$ & 67.472 & 9 & 0.56 & 10 & 0.60 \\
60 & $\mathbf{5}$ & 63.706 & 10 & 0.50 & 11 & 0.55 \\
60 & $\mathbf{6}$ & 60.890 & 11 & 0.45 & 11 & 0.45 \\
60 & $\mathbf{7}$ & 58.316 & 12 & 0.42 & 12 & 0.42 \\
60 & $\mathbf{8}$ & 56.143 & 12 & 0.33 & 13 & 0.38 \\
60 & $\mathbf{9}$ & 54.238 & 13 & 0.31 & 14 & 0.36 \\
60 & $\mathbf{1 0}$ & 52.596 & 14 & 0.29 & 15 & 0.33 \\
60 & $\mathbf{2 0}$ & 40.032 & 22 & 0.09 & 22 & 0.09 \\
\hline
\end{tabular}

\begin{tabular}{|l|c|c|l|l|c|l|}
\hline \multicolumn{2}{|l|}{ Reference: } & \multicolumn{3}{l|}{ Equivalent $n$ to produce $\tilde{Z}$} \\
\cline { 4 - 7 } BW1 & \multicolumn{2}{|l|}{ BW2 } & \multicolumn{2}{l|}{ MLE } \\
\hline$m$ & $n$ & $\tilde{Z}$ & $n$ & \% Saving & $n$ & $\%$ Saving \\
\hline 80 & $\mathbf{3}$ & 70.342 & 14 & 0.79 & 15 & 0.80 \\
80 & $\mathbf{4}$ & 64.329 & 16 & 0.75 & 17 & 0.76 \\
80 & $\mathbf{5}$ & 60.164 & 18 & 0.72 & 19 & 0.74 \\
80 & $\mathbf{6}$ & 56.752 & 19 & 0.68 & 21 & 0.71 \\
80 & $\mathbf{7}$ & 53.973 & 21 & 0.67 & 22 & 0.68 \\
80 & $\mathbf{8}$ & 51.766 & 22 & 0.64 & 23 & 0.65 \\
80 & $\mathbf{9}$ & 50.060 & 22 & 0.59 & 24 & 0.63 \\
80 & $\mathbf{1 0}$ & 48.616 & 23 & 0.57 & 24 & 0.58 \\
80 & $\mathbf{2 0}$ & 40.194 & 27 & 0.26 & 28 & 0.29 \\
\hline
\end{tabular}

Figure 5 plots median confidence intervals of the methods for $m=1.5,10$ and 80, that is, any lower or upper bound in the plots is the median of the lower or upper bounds of 10,000 confidence intervals estimated by Monte Carlo simulations. They show that the BW2 model generates confidence intervals that are more 
centered around $m$ as compared to the MLE and the BW1. This phenomenon is particularly noticeable for small samples.

a)

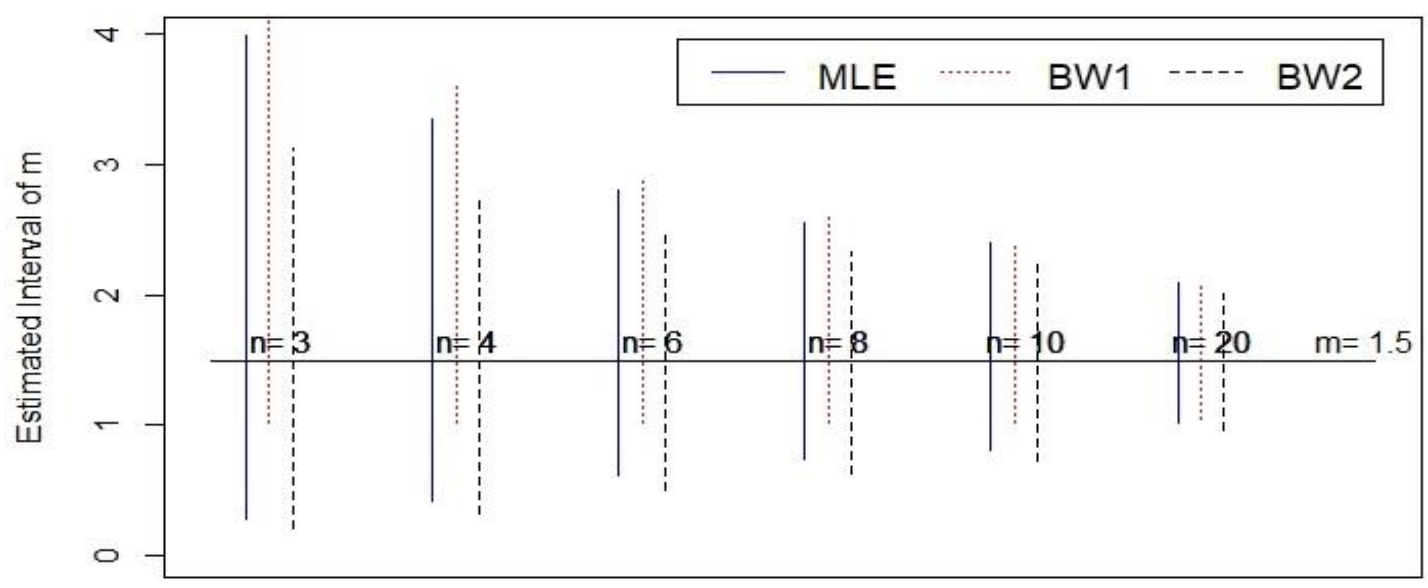

b)

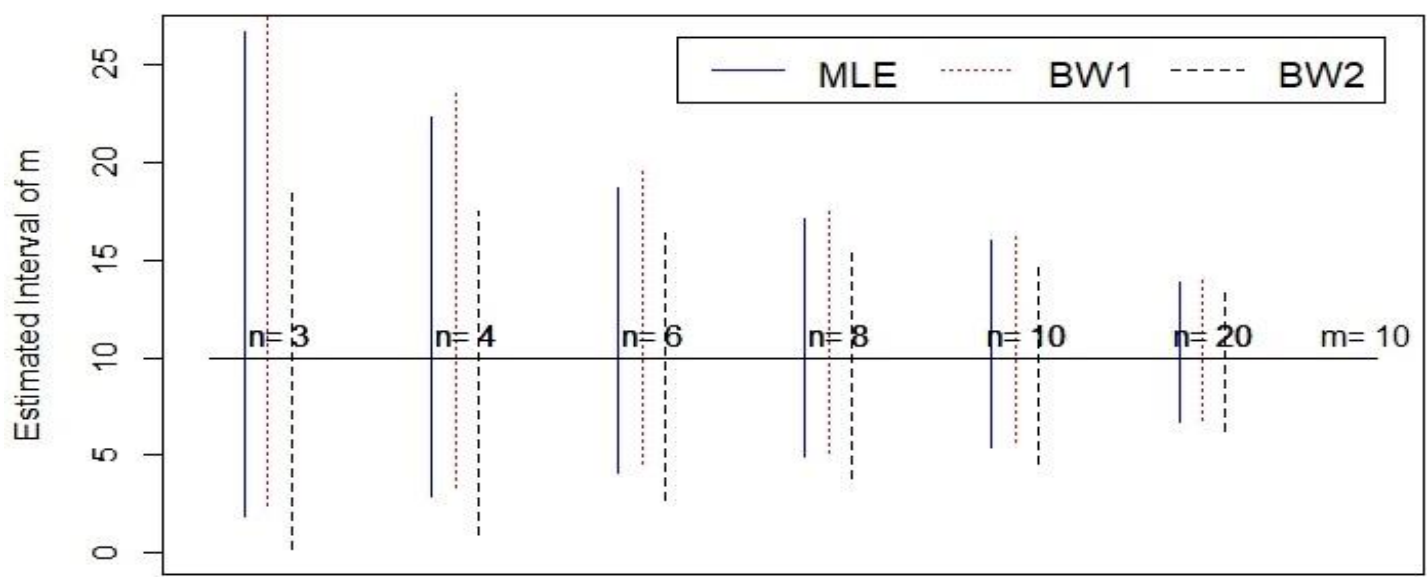

c)

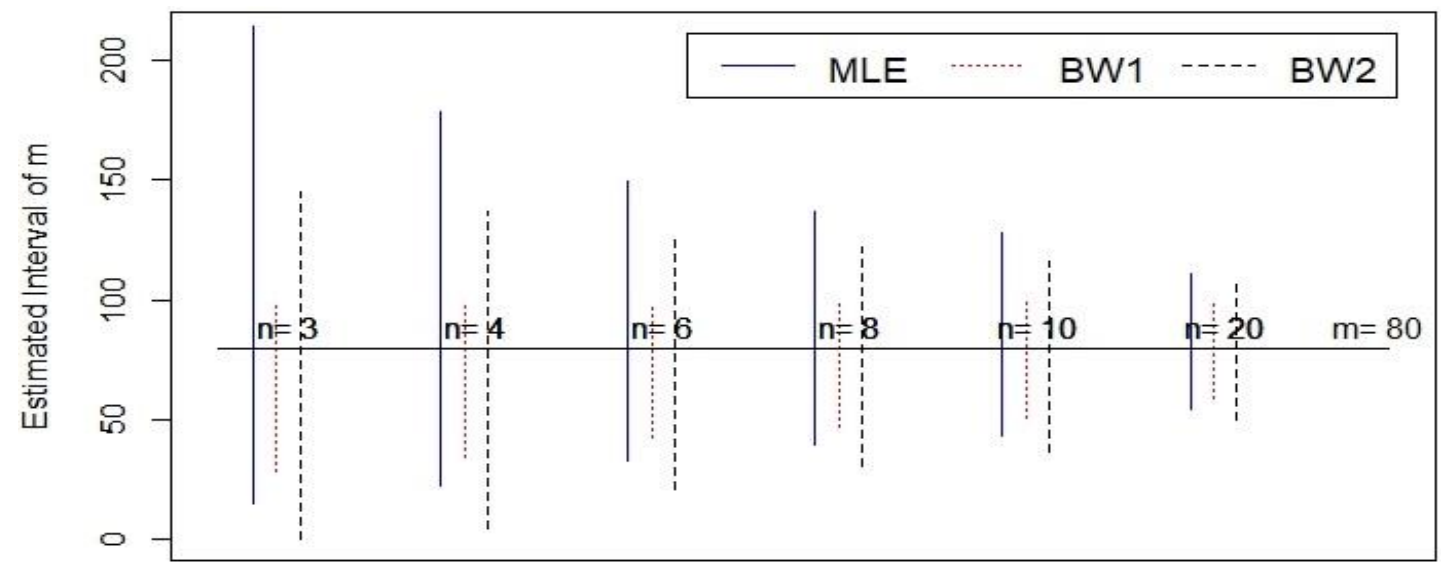

Figure 5. The position of estimated confidence intervals according to a) $m=1.5 \mathrm{~b}$ ) $m=10$ and c) $m=80$

\section{VERIFICATION OF THE SIMULATION}

Verification of the simulation results was carried out by an experimental study cited in the literature [73]. The experimental study was on the fracture strengths of 19 identical composite specimens. The composite specimens were prepared from quasi-isotropic carbon- epoxy sheets with $\left(0^{\circ}\right)_{3}$ configuration, $0.89 \mathrm{~mm}$ thickness, and $295 \mathrm{~g} / \mathrm{m}^{2}$ weight. The tension experiments were carried out on an Instron 8516+universal testing machine according to ASTM D3039 standard [73]. A crosshead speed of $1.33 \mathrm{~mm} / \mathrm{min}$ was used and room temperature conditions existed during the tests. The fracture strength values measured are presented in Table 5. 
\begin{tabular}{llllllllll} 
Table 5. Fracture strength values from tension experiments & \\
\hline $\mathbf{1}$ & $\mathbf{2}$ & $\mathbf{3}$ & $\mathbf{4}$ & $\mathbf{5}$ & $\mathbf{6}$ & $\mathbf{7}$ & $\mathbf{8}$ & $\mathbf{9}$ & $\mathbf{1 0}$
\end{tabular}

\begin{tabular}{lcccccccccc}
\hline Test No & $\mathbf{1}$ & $\mathbf{2}$ & $\mathbf{3}$ & $\mathbf{4}$ & $\mathbf{5}$ & $\mathbf{6}$ & $\mathbf{7}$ & $\mathbf{8}$ & $\mathbf{9}$ & $\mathbf{1 0}$ \\
Fracture strength & & & & & & & & & & \\
(MPa) & 532.7 & 502.5 & 442 & 473 & 519 & 502.7 & 477 & 510 & 522 & 552 \\
Test No & $\mathbf{1 1}$ & $\mathbf{1 2}$ & $\mathbf{1 3}$ & $\mathbf{1 4}$ & $\mathbf{1 5}$ & $\mathbf{1 6}$ & $\mathbf{1 7}$ & $\mathbf{1 8}$ & $\mathbf{1 9}$ & \\
$\begin{array}{l}\text { Fracture strength } \\
\text { (MPa) }\end{array}$ & 522 & 439 & 513.6 & 497.5 & 521.6 & 450.9 & 476.5 & 507.3 & 463.5 & \\
\hline
\end{tabular}

In order to diversify the application study in terms of sample size, besides the entire sample, the random subsets of size of 3,5 and 10 were drawn from the sample of 19 observations. The data set consists of $\{513.6,442,519\}$ for $\mathrm{n}=3,\{510,521.6,442,439,519\}$ for $\mathrm{n}=5$ and $\{439,521.6,513.6,450.9,442,463.5$, $473,519,476.5,477\}$ for $n=10$. By the three methods, the confidence intervals of $m$ were estimated and the result of the best method for each $n$ was shown in bold in Table 6 .

Table 6. The experimental results for $n=3,5,10$ and 19

\begin{tabular}{|c|c|c|c|c|}
\hline \multirow{3}{*}{$\boldsymbol{n}$} & \multirow{2}{*}{ Method } & \multicolumn{3}{|c|}{ Confidence Interval of $\boldsymbol{m}(\boldsymbol{\alpha}=\mathbf{0 . 0 5})$} \\
\cline { 3 - 5 } & & Lower Bound & Upper Bound & Interval Length \\
\hline \multirow{3}{*}{3} & MB & 2.463 & 35.720 & 33.257 \\
\cline { 2 - 5 } & BW1 & 2.919 & 41.927 & 39.008 \\
\cline { 2 - 5 } & BW2 & $\mathbf{0 . 4 0 9}$ & $\mathbf{2 5 . 7 7 4}$ & $\mathbf{2 5 . 3 6 5}$ \\
\hline \multirow{3}{*}{5} & MB & 4.890 & 27.463 & 22.573 \\
\cline { 2 - 5 } & BW1 & 5.014 & 30.329 & 25.315 \\
\cline { 2 - 5 } & BW2 & $\mathbf{2 . 2 5 6}$ & $\mathbf{2 3 . 4 9 0}$ & $\mathbf{2 1 . 2 3 4}$ \\
\hline \multirow{3}{*}{10} & MB & 8.536 & 25.191 & 16.655 \\
\cline { 2 - 5 } & BW1 & 9.091 & 25.595 & 16.504 \\
\cline { 2 - 5 } & BW2 & $\mathbf{7 . 4 4 2}$ & $\mathbf{2 3 . 3 1 8}$ & $\mathbf{1 5 . 8 7 6}$ \\
\hline \multirow{3}{*}{19} & MB & 11.980 & 25.132 & 13.152 \\
\cline { 2 - 5 } & BW1 & 12.162 & 25.205 & $\mathbf{1 2 . 8 5}$ \\
\cline { 2 - 5 } & BW2 & $\mathbf{1 1 . 1 7 1}$ & $\mathbf{2 4 . 0 2 1}$ & \\
\hline
\end{tabular}

As can be seen in Table 6, the experimental results are quite similar to simulation results and the BW2 model outperforms the other methods. Especially for $n=3$, it yields a much narrower interval than those of the MLE and BW1. As $n$ increases, performances of the methods become closer to each other, but the BW2 model maintains its superiority by a small difference in estimated interval length.

\section{RESULTS}

This study compares the performances of the two proposed Bayesian models against the Maximum Likelihood Method (MLE) for estimating confidence intervals of the Weibull modulus in small samples. The first Bayesian model (BW1) assumes that the Weibull modulus has a uniform prior on the interval of $(1,100)$. The second Bayesian model (BW2) assumes that the Weibull modulus is between 1 and 100 with a probability, $1-\alpha$. Non-informative priors are used for the scale parameter.

In the BW1 model, use of uniform prior on the interval of $(1,100)$ was recently proposed in the materials science literature, and the lower and uppers limits, 1 and 100, was supported by practical and theoretical studies in the literature. The BW2 model is a relaxed version of the BW1 model, assigning a high probability $(1-\alpha)$ to the event that Weibull modulus is in this interval, instead of requiring it to be in the interval with a probability of 1 , which is the case in the BW1 model.

The Weibull modulus values between 1.5 and 80, and sample size values between 3 and 20 are considered in this study, and the simulations showed that the Bayesian models always have better performance than the MLE method. In general, the BW1 model is the best method for the Weibull modulus values close to 1 
and 100, and the BW2 model is the best for the other values between 1 and 100 , particularly for $2 \leq m \leq 40$. The performances of the Bayesian models become better as the sample size decreases, particularly for $n \leq 10$, which is an expected result from the general Bayesian theory. In addition, the BW2 model generates confidence intervals that are more centered around $m$. This result is important for engineers, because they usually expect the unknown parameter to lie at or near the center of the estimated interval.

In summary, this study proposes the BW2 model for estimating the confidence (credible) interval of Weibull modulus, especially for small sample sizes since the BW2 model has more relaxed assumptions than the BW1 model, and performs better unless the Weibull modulus values close to 1 and 100 .

There are many alternative Bayesian methods that can be used for Weibull applications in materials science such as recursive methods. This study demonstrates that Bayesian methods have a promising future in reliability studies in materials science, because, they attempt to capture the existing domain knowledge, such as increasing failure rates, and reflect it in the estimation process. This results in higher estimation precision.

A final word of caution is that while using priors based on unfounded assumptions and non-existing knowledge may result in Bayesian methods with superior performances, the obtained results would likely be non-representative of the domain and misleading. In Bayesian analysis, priors should reflect the true domain knowledge. In this study, care has been taken to ensure compliance to this general rule.

\section{CONFLICTS OF INTEREST}

No conflict of interest was declared by the authors.

\section{REFERENCES}

[1] Weibull, W., "A Statistical Theory of the Strength of Materials". Ingvetenskaps Akad. Handl. 151, Stockholm (1939).

[2] Barbero, E., Fernández-Sáez, J. and Navarro, C., "Statistical analysis of the mechanical properties of composite materials", Compos. Part B-Eng, 31(5): 375-381, (2000).

[3] McCool, J., "Flexural strength tests of brittle materials: selecting the number of specimens and determining confidence limits for Weibull parameters", J. Test Eval., 45(2): 664-670 (2016).

[4] Barbero, E., Fernández-Sáez, J. and Navarro, C., "Statistical distribution of the estimator of Weibull modulus", J. Mater. Sci. Lett., 20(9): 847-849, (2001).

[5] McCool, J.I., Using the Weibull distribution: Reliability, Modeling, and Inference, 1st ed. NJ: John Wiley \& Sons Inc, (2012).

[6] Wua, D., Lia, Y., Zhanga, J., Changa, L., Wu, D. ,Fang, Z. and Shic, Y., "Effects of the number of testing specimens and the estimation methods on the Weibull parameters of solid catalysts", Chem. Eng. Sci., 56(24): 7035-7044, (2001).

[7] Bao, Y.W. and Zhou, Y., "Investigation on Reliability of Nanolayer Grained Ti3SiC2 via Weibull Statistics", J. Mater. Sci., 42(12): 4470-4475, (2007).

[8] Nohut, S., "Influence of sample size on strength distribution of advanced ceramics", Ceram. Int., 40(3): 4285-4295, (2014).

[9] Khalili, A. and Kromp, K., "Statistical properties of Weibull estimators", J. Mater. Sci., 26(24): 67416752, (1991). 
[10] Durham, S., Lynch, J. and Padgett, W., "Inference for strength distributions of brittle fibers under increasing failure rate", J. Composite Mater., 22(12): 1131-1140, (1988).

[11] Durham, S., Lynch, J. and Padgett, W., "A theoretical justification for an increasing average failure rate strength distribution in fibrous composites", Naval Res. Logistics (NRL), 36(5): 655-661, (1989).

[12] Durham, S., Lynch, J. and Padgett, W., "TP 2-orderings and the IFR property with applications", Probability Eng. Inf. Sci., 4(01): 73-88, (1990).

[13] Yalcinkaya, M. and Birgoren, B., "Confidence interval estimation of Weibull lower percentiles in small samples via Bayesian inference”, J. Eur. Ceram. Soc., 37: 2983-2990, (2017).

[14] Meyers, M.A. and Chawla, K.K., Mechanical Behavior of Materials, Cambridge: Cambridge University Press, (2009).

[15] Askeland, D.R., Fulay, P. and Wright, W., The Science and Engineering of Materials, 6th ed. Starnford: Thomson Learning Inc, (2010).

[16] Bousquet, N., "Eliciting vague but proper maximal entropy priors in Bayesian experiments", Stat Pap., 51(3): 613-628, (2010).

[17] Abernethy, R.B., The New Weibull Handbook, 5th ed. North Palm Beach: Fla.R.B. Abernethy, (2006).

[18] Rinne, H., The Weibull Distribution: A Handbook, FL: CRC Press, (2009).

[19] Dodson, B., The Weibull Analysis Handbook, 2nd ed. Milwaukee: ASQ Quality Press, (2006).

[20] Ambrožič, M. and Gorjan, L., "Reliability of a Weibull analysis using the maximum-likelihood method", J. Mater. Sci., 46(6): 1862-1869, (2011).

[21] Davies, I.J., "Unbiased estimation of Weibull modulus using linear least squares analysis-A systematic approach”, J. Eur. Ceram. Soc., 37(1): 369-380, (2017).

[22] Bergman, B., "Estimation of Weibull parameters using a weight function", J. Mater. Sci. Lett., 5(6): 611-614, (1986).

[23] Faucher, B. and Tyson, W., "On the determination of Weibull parameters", J. Mater. Sci. Lett., 7(11): 1199-1203, (1988).

[24] Birgoren, B., Ceramics and Composite Materials: New Research, New York : Nova Science Publishers, 215-235, (2006).

[25] Nelson, W., Applied Life Data Analysis, New York: John Wiley \& Sons, (2005).

[26] Johnson, N.L., Kotz, S. and Balakrishnan, N., Continuous Univariate Distributions, 2nd ed. New York: Wiley, (1995).

[27] Murthy, D.N.P, Xie, M. and Jiang, R., Weibull Models, New York: Wiley, (2004).

[28] Langlois, R., "Estimation of Weibull parameters”, J. Mater. Sci. Lett., 10(18): 1049-1051, (1991).

[29] Gurvich, M., Dibenedetto, A. and Pegoretti, A., "Evaluation of the statistical parameters of a Weibull distribution", J. Mater. Sci., 32(14): 3711-3716, (1997). 
[30] Gong, J., "Determining the confidence intervals for Weibull estimators", J. Mater. Sci. Lett., 18(17): 1405-1407, (1999).

[31] Gong, J., "A new probability index for estimating Weibull modulus for ceramics with the least-square method", J. Mater. Sci. Lett., 19(10): 827-829, (2000).

[32] Davies, I., "Empirical correction factor for the best estimate of Weibull modulus obtained using linear least squares analysis", J. Mater. Sci. Lett., 20(11): 997-999, (2001).

[33] Davies, I., "Best estimate of Weibull modulus obtained using linear least squares analysis: an improved empirical correction factor", J. Mater. Sci. Lett., 39(4): 1441-1444, (2004).

[34] Griggs, J. and Zhang, Y., "Determining the confidence intervals of Weibull parameters estimated using a more precise probability estimator”, J. Mater. Sci. Lett., 22(24): 1771-1773, (2003).

[35] Wu, D. and Jiang, H., "Comment on "A new probability index for estimating Weibull modulus for ceramics with the least-square method", J. Mater. Sci. Lett., 22(24):1745-1746, (2003).

[36] Tiryakioğlu, M., "On estimating Weibull modulus by moments and maximum likelihood methods", J. Mater. Sci., 43(2): 793-798, (2008).

[37] Tiryakioğlu, M., Hudak, D., "Guidelines for two-parameter Weibull analysis for flaw-containing materials", Metall. Mater. Trans. B., 42(6): 1130-5, (2011).

[38] Phan, L. and McCool, J., "Exact confidence intervals for Weibull parameters and percentiles", P. I. Mech. Eng. O-J Ris., 223(4): 387-394, (2009).

[39] Menon, M.V., "Estimation of the shape and scale parameters of the Weibull distribution", Technometrics, 5: 175-182, (1963).

[40] McCool, J.I., "Software for Weibull inference”, Quality Engineering, 23: 253-264, (2011).

[41] Bütikofera, L., Stawarczykb, B. and Roosa, M., "Two regression methods for estimation of a twoparameter Weibull distribution for reliability of dental materials", Dent. Mater., 31: 33-50, (2015).

[42] Ahmed, A.O.M., Ibrahim, N.A. and Al-Kutubi, H.S., "Comparison of the Bayesian and maximum likelihood estimation for Weibull distribution”, J. Math. Stat., 6(2): 100-104, (2010).

[43] Alkutubi, H.S., AlShemmary E.N.A., Yasseen S.A. and Alwan Y.H., "New bayes estimator of parameter Weibull distribution using simulation study", IJBAS, 1(3): 237-243, (2012).

[44] Aslam, M., Kazmi, S.M.A. and Ahmad, I., "Bayesian estimation for parameters of the Weibull distribution”, Sci.Int.(Lahore), 27(1): 259-264, (2014).

[45] Guure, C.B., Ibrahim, N.A. and Ahmed, A.O.M., "Bayesian estimation of two-parameter Weibull distribution using extension of Jeffreys' prior information with three loss functions", Math. Probl. Eng., 2012(13), (2012).

[46] Guure, C.B. and Ibrahim, N.A., "Approximate Bayesian estimates of Weibull parameters with Lindley's method”, Sains Malaysiana, 43(9): 1433-1437, (2014).

[47] Jia, J., Yan, Z. and Peng, X., “A New Discrete Extended Weibull Distribution”, IEEE Access , 7: 175474- 175486, (2019). 
[48] Aron, A., Guo, H., Mettas, A. and Ogden D., "Improving the 1-parameter weibull: A bayesian approach", Annual Reliability and Maintainability Symposium, 432-435, (2009).

[49] http://reliawiki.com/index.php/The_Weibull_Distribution. Access date: 13.05.2020.

[50] Simoa, N.F., Wieler, M., Feltenb, F. and Reh S., "Bayesian analysis for determination and uncertainty assessment of strength and crack growth parameters of brittle materials", J. Eur. Ceram. Soc., 37: 17691777, (2017).

[51] Nguyen, D.L. Thai, D.K., Ngo, T.T, Tran, T.K. and Nguyen, T.T., "Weibull modulus from size effect of high-performance fiber-reinforced concrete under compression and flexure", Constr. Build. Mater., 226 (30): 743-758, (2019).

[52] Ono, K., "A Simple Estimation Method of Weibull Modulus and Verification with Strength Data", Appl. Sci., 9(8): 1575, (2019)

[53] Zellner, A., New developments in the applications of Bayesian methods, Amsterdam:University of Chicago Press, 211-232, (1977).

[54] Zellner, A., Maximum entropy and Bayesian methods, Boston: Kluwer, 17-31, (1991).

[55] Zellner, A., "Models, prior information and Bayesian analysis", J. Econom., 75: 51-68, (1996).

[56] Berger, J.O., Statistical decision theory and Bayesian analysis, 2nd ed. New York: Springer, (2013).

[57] Soofi, E.S., Bayesian Analysis in Statistics and Econometrics, D.A. Berry, K.M. Chaloner, J.K. Geweke (eds).New York: Wiley, (1992).

[58] Skilling, J., Maximum entropy and Bayesian methods, Dordrecht:Kluwer Academic Publisher, (1989).

[59] LeBesnerais, G., Bercher, J.F. and Demoment, G., "A new look at entropy for solving linear inverse problems", IEEE Trans. Inform. Theory, 45: 1565-1578, (1999).

[60] Soofi, E.S., "Principal information theoretic approaches”, J. Am. Stat. Assoc., 95: 1349-1353, (2000).

[61] Miller, D.J. and Yan, L., "Approximate maximum entropy joint feature inference consistent with arbitrary lower order probability constraints: application to statistical classification", Neural Comput., 12: 2175-2208, (2000).

[62] Green, N.R. and Campbell J., Mater. Sci. Eng. A, A137: 261-266, (1993).

[63] Ćurković, L., Bakić, A., Kodvanj, J. and Haramina, T., "Flexural strength of alumina ceramics: Weibull analysis", Transactions of FAMENA, 34(1): 13-19, (2010).

[64] Askeland, D.R. and Fulay, P.P., Essentials of Materials Science and Engineering, 3nd ed., Canada: Cengage Learning, (2013).

[65] Tobias, P.A. and Trindade, D., Applied Reliability. 3nd ed., Boca Raton: CRC Press, (2011).

[66] Birgoren, B. and Dirikolu, M.H., "A computer simulation for estimating lower bound fracture strength of composites using Weibull distribution”, Compos. Part B-Eng., 35(3): 263-266, (2004).

[67] Thoman, D.R., Bain L.J. and Antle C.E., "Inferences on the parameters of theWeibull distribution", Technometrics, 11: 445-460, (1969). 
[68] Suen, H.K., Principles of Test Theories, New York: Routledge, (2012).

[69] Glasgow, L.A., Applied Mathematics for Science and Engineering, John Wiley \& Sons, (2014).

[70] Casella, G. and Berger, R.L., Statistical Inference, 2nd ed. USA: Thomson Learning, (2002).

[71] Riedel, R. and Chen, I.W., Ceramics Science and Technology, John Wiley \& Sons, (2011).

[72] Juritz, J. M., Juritz, J. W. F. and Stephens, M. A., "On the accuracy of simulated percentage points", J. Am. Stat. Assoc., 83: 441-444, (1983).

[73] Dirikolu, M.H., Aktas, A. and Birgören, B., "Statistical analysis of fracture strength of composite materials using Weibull distribution", Turkish J Engng Environ Sci, 26(1): 45-8, (2002). 